\title{
Primary thyroid teratoma in adults: A case report and systematic review of the literature
}

\author{
CONSTANTINOS NASTOS $^{1}$, ANNA PASPALA ${ }^{1}$, MATHEW STAMELOS ${ }^{1}$, IOANNA MAVROEIDI $^{2}$, \\ KONSTANTINOS PROIKAS ${ }^{3}$, GEORGIA THOMOPOULOU ${ }^{4}$, AMANDA PSYRRI $^{5}$ and EMMANUIL PIKOULIS ${ }^{1}$ \\ ${ }^{1}$ Third Department of Surgery, Attikon University Hospital; ${ }^{2}$ Endocrine Unit, \\ 2nd Propaedeutic Department of Internal Medicine and Research Institute, Attikon University Hospital; \\ ${ }^{3}$ ENT Department, Attikon University Hospital; ${ }^{4}$ Department of Cytopathology, Attikon University Hospital; \\ ${ }^{5}$ Department of Internal Medicine, Section of Medical Oncology, School of Medicine, Attikon University Hospital, \\ National and Kapodistrian University of Athens, 12462 Athens, Greece
}

Received December 30, 2020; Accepted April 8, 2021

DOI: $10.3892 /$ mco.2021.2331

\begin{abstract}
Extragonadal germ cell tumors are uncommon in adults and only $2-5 \%$ of teratomas develop in extragonadal sites. Primary thyroid teratomas represent $<0.1 \%$ of all primary thyroid gland neoplasms. In the present report, a case of primary thyroid teratoma in a 65-year-old female is described. Furthermore, the current literature regarding patients who were diagnosed with primary thyroid teratoma and underwent surgical resection was systematically reviewed. A total of 15 studies of 27 patients (age range, 17-65 years). Growing mass or neck swelling were the primary symptoms in 14 patients $(51.8 \%)$. Only one $(5.5 \%)$ patient was preoperatively diagnosed with malignant thyroid teratoma. All patients underwent thyroidectomy, but 6 cases had more advanced surgery, including lymph node dissection. A total of 12 patients received a combination of adjuvant chemoradiation postoperatively, $10(45.4 \%)$ patients reported recurrence of disease and 8 (29.6\%) were postoperatively diagnosed with distant metastases. A total of 9 (39.1\%) patients died due to progression of the disease. In conclusion, primary thyroid teratomas are rare and difficult to diagnose preoperatively. In particular, malignant cases are very aggressive tumors with a considerably poor prognosis, even after surgical resection combined with adjuvant chemoradiation.
\end{abstract}

\section{Introduction}

Extragonadal germ cells tumors are uncommon in adults and only $2-5 \%$ of teratomas can develop in extragonadal sites $(1,2)$. The most frequent extragonadal teratoma locations are anterior

Correspondence to: Dr Anna Paspala, Third Department of Surgery, Attikon University Hospital, National and Kapodistrian University of Athens, 1 Rimini Street, 12462 Athens, Greece E-mail: garoufalo@hotmail.com

Key words: thyroid teratoma, thyroidectomy, extragonadal, malignant thyroid neoplasm mediastinum and retroperitoneum in almost, occurring 60 and $40 \%$ of cases, respectively (1). However, the proportion of head and neck teratomas is approximately 6\% (3-5).

Primary Thyroid Teratomas (PTTs) are rare thyroid neoplasms of germ cells derivation that are characterized by features of trilineage differentiation. PTTs are commonly benign, especially, in infants and children; however, they are mostly malignant in adults (6). Females are more frequently diagnosed with PTT than men. Typical clinical manifestations of PTTs are neck mass and neck swelling accompanied by lymphadenopathy, while malignant PTTs can develop distant metastases; the most common locations of metastatic disease are lungs and bones $(7,8)$. According to the grading criteria of gonadal teratomas, PTTs are categorized in three groups: Benign (grade 0), immature (grade 1 and 2) and malignant (immature grade 3) teratomas (2). Malignant teratomas affect almost only adults and can occur with a significantly more aggressive clinical behavior compared with benign and immature teratomas (9).

In the majority of cases, surgical resection is the primary treatment of choice for PTT. However, the introduction of chemotherapy as a postoperative adjuvant therapy has produced some revolutionary outcomes in prognosis and overall survival (10). Although benign PTTs have an excellent long-term prognosis, malignant PTTs are characterized by overall survival rates even less than 12 months $(6,7)$. Recurrence of disease, either in the thyroid bed or regional neck lymph nodes, and the development of metastatic disease are commonly associated with decreased survival rates and higher rates of cancer-related deaths (7).

To that end, in the present study, an individual case report of a patient with PTT and a systematic review of the literature. We analyzed the clinical characteristics, perioperative management and the long-term postoperative and oncological outcomes after surgical resection in patients diagnosed with PTT.

\section{Case report}

A 65-year-old female presented to our clinic with a one-month history of a neck mass. No pertinent past medical illness or family history was present. On physical examination, a large hard and 
mobile mass was palpated in the right thyroid lobe. The laboratory findings were unremarkable, and her thyroid function was normal The Ultrasound (US) scan of neck revealed a $5.5 \times 3.5 \mathrm{~cm}$ solid nodule in the right lobe of thyroid. Fine needle aspiration (FNA) of thyroid nodule was performed by the Endocrine surgeons of our Unit under US guidance, and the diagnosis of papillary thyroid carcinoma was then suggested (Bethesda VI). As a next diagnostic step, a detailed US neck of central and lateral compartments was performed, and a $3 \mathrm{~cm}$ lymph node was detected in the right cervical compartment. Lymph's node FNA was negative for malignancy. The patient was scheduled for total thyroidectomy with central neck dissection. Intraoperatively, the tumor was penetrating the thyroid capsule and was invading the adjacent tissue involving the right recurrent laryngeal nerve, thus, a small part of thyroid tissue surrounding the right recurrent laryngeal nerve was not excised. Frozen section was not performed. The postoperative period was uneventful, except for a diagnosis of asymptomatic hypocalcemia. The patient was discharged on 1st postoperative day with oral calcium supplementation. Histopathological examination of the surgical specimen revealed a $5.5 \mathrm{~cm}$ malignant PTT (grade 3) of the right thyroid lobe with heterogenous characteristics and a synchronous $0.5 \mathrm{~cm}$ PTC of left thyroid lobe. Immunohistochemical studies showed positive staining of PTT's cells CD56, Glypican3 and TTF-1 and negative staining for Synaptophysin, Chromogranin, CD45, CD30 and S-100. Regarding the enlarged cervical lymph node, the histology was negative for malignancy. Four weeks after the patient's first operation, she presented at our emergency department with acute airway obstruction. She urgently underwent surgery for tracheostomy. A computed tomography scan after the tracheostomy showed a considerable progression of disease in neck (Fig. 1). The patient is still alive and has been admitted in the Oncology Unit of our hospital, where she received a cis-platinum based chemotherapy.

\section{Literature review}

We systematically searched the literature for articles published from January 2000 up to September 2020 using PubMed (1966-2019), Scopus (2004-2019) and Google Scholar (2004-2019) databases along with the references of the articles, which were retrieved in full text. The following key words were used for the search: 'Thyroid teratoma' 'primary thyroid teratoma' 'thyroidectomy' and 'malignant thyroid teratoma'. A minimum number of key words were utilized in order to assess an eligible number that could be easily searched while simultaneously minimizing the potential loss of articles. Articles that fulfilled or were deemed to fulfil the inclusion criteria were retrieved. All articles published from January 2000 which reported cases of patients aged $>16$ years who underwent thyroidectomy and/or neck dissection for primary thyroid teratoma were included. Data on patient characteristics included age and sex, while disease characteristics included primary symptoms and diagnosis, preoperative imaging studies, size of tumor and type of surgery. Concerning the main findings of the study, histopathological outcomes, survival rates regarding incidence of recurrence and cancer-related deaths were also assessed. The Preferred Reporting Items for Systematic Reviews and Meta-analyses (PRISMA) flow diagram schematically presents the stages of article selection (Fig. 2).

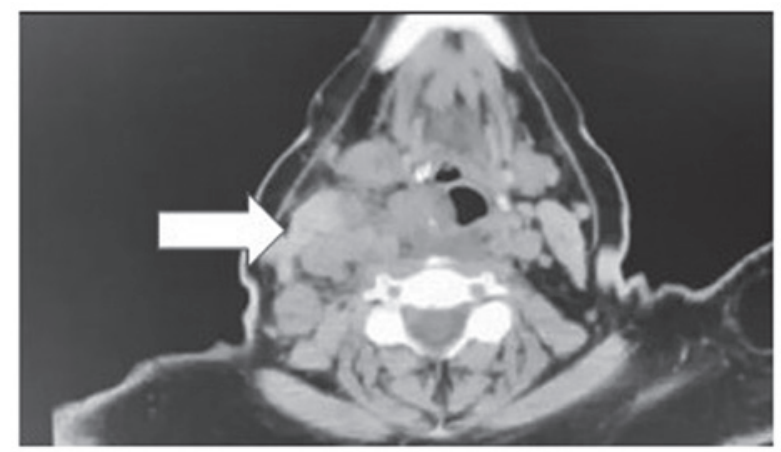

Figure 1. Postoperative CT image. A postoperative neck CT revealed a large recurrent neck mass (white arrow), which resulted in an airway obstruction that required an urgent tracheostomy. CT, computed tomography.

A total of 15 studies (11 case reports and 4 case series) described outcomes for 27 patients who were diagnosed with PTT and underwent thyroidectomy and in some cases an additional neck dissection $(1,2,4,8,9,11-20)$. One study was excluded from the present systematic review (6). The study conducted by Thompson et al was excluded due to insufficient specific data concerning patients' main characteristics and survival outcomes (6).

Table I illustrates the main characteristics of the 27 patients reviewed. The proportion of men was $18.5 \%$ in the current review, while the ages ranged from 17 to 65 years. As shown in Table I, primary symptoms and signs were described in all included cases. A growing mass or neck swelling were the primary sign in $14(51.8 \%)$ patients, while odynophagia was observed in $4(14.8 \%)$ patients. Four $(14.8 \%)$ patients presented with other symptoms $(4,11,17,18)$. Of these, one (3.7\%) presented with severe airway obstruction, one $(3.7 \%)$ with dysphonia, one $(3.7 \%)$ with vocal cord paralysis and one $(3.7 \%)$ case with hoarseness of voice. Preoperative imaging studies were reported in $77.7 \%$ of cases $(n=21$ out of 27 patients), included a combination of neck Ultrasound (US), Sestamibi scan, Computed Tomography (CT), Positron Emission Tomography (PET) and Fine-needle aspiration (FNA) biopsy are presented in Table I. More specifically, the percentage of patients who had a preoperative neck US and CT for assessing nodules of thyroid gland were 66.6 and $57.1 \%$, respectively. PET scan performed preoperatively in 3 out of 21 patients $(14.2 \%)$, whereas only 2 cases had a Sestamibi scan $(9.5 \%)(2,8,13,16,18)$. Moreover, FNA biopsy of suspicious lesions of thyroid gland was used in approximately $90.4 \%$ ( $n=19$ out of 21 patients) of included patients.

A specific preoperative diagnosis was recorded in 18 (66.6\%) cases. Among them, 4 (22.2\%) patients diagnosed with medullary thyroid carcinoma, whereas poorly differentiated thyroid carcinoma and undifferentiated thyroid carcinoma was reported in 3 $(16.6 \%)$ and $2(11.1 \%)$ patients, respectively. Additionally, follicular thyroid neoplasm was diagnosed in $3(16.6 \%)$ cases, while one $(5.5 \%)$ patient was diagnosed with hurthle cell neoplasm and one $(5.5 \%)$ diagnosed with high-grade sarcoma, preoperatively. As a preoperative diagnosis, FNA biopsy reported malignant tumor and colloid nodule with cellular atypia in 1 (5.5\%) case, respectively $(4,16)$. One case $(5.5 \%)$ had a preoperative diagnosis of a tumor with neuroepithelial differentiation (20). Only one (5.5\%) patient was diagnosed with malignant thyroid teratoma, 


\section{PRISMA 2009 Flow Diagram}

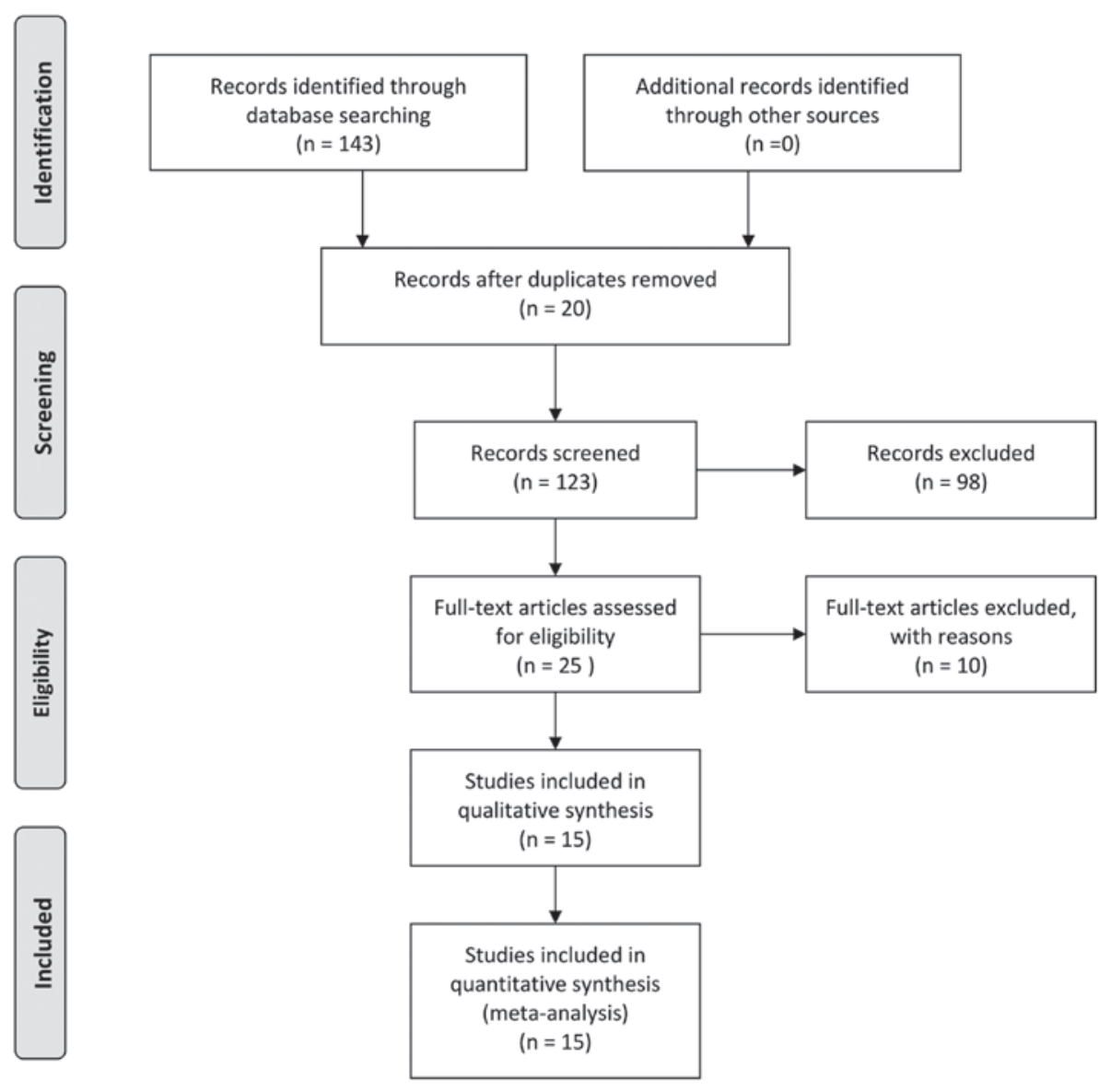

From: Moher D, Liberati A, Tetzlaff J, Altman DG, The PRISMA Group (2009). Preferred Reporting hems for Systematic Reviews and MetaAnalyses: The PRISMA Statement. PLoS Med 6(7): e1000097. doi:10.1371/journal.pmed1000097

For more information, visit www.prisma-statement.org.

Figure 2. PRISMA flow diagram. The diagram presents the process of article selection. PRISMA, Preferred Reporting Items for Systematic Reviews and Meta-analyses.

preoperatively (2). The median size of thyroid lesions from preoperative studies was $6.1 \mathrm{~cm}$.

Operative outcomes. All patients underwent surgery for PTT. The specific technique was described in 18 (66.6\%) patients. Among them, 5 (27.7\%) underwent total thyroidectomy, 4 (22.2\%) subtotal thyroidectomy and $3(16.6 \%)$ lobectomies. Additionally, $6(33.3 \%)$ patients underwent a more complicated surgical approach due to a considerably advanced disease $(2,11-13,17,20)$. Of these cases, 2 (33.3\%) underwent total thyroidectomy and central neck dissection, $2(33.3 \%)$ had total thyroidectomy combined with bilateral modified neck dissection, one (16.6\%) underwent total thyroidectomy with additional central and left lateral neck dissection, and one $(16.6 \%)$ had total thyroidectomy plus left cervical and mediastinal lymph node dissection. Only one (16.6\%) patient needed additional tracheostomy during subtotal thyroidectomy due to advanced disease (2). Histopathological examination was reported in all cases. Only one (3.7\%) patient was diagnosed with a primary benign thyroid teratoma (15), whereas 26 cases were diagnosed with primary malignant thyroid teratoma. Metastatic disease in cervical lymph nodes was reported in approximately $44.4 \%$ of the included patients ( $n=12$ out of 27 cases).

Postoperative and oncological outcomes. Twelve (44.4\%) patients received a combination of adjuvant chemoradiation postoperatively. Eight (29.6\%) patients received only adjuvant chemotherapy. After surgery, 4 (14.8\%) cases received no adjuvant therapy, whereas interestingly $2(7.4 \%)$ patients had cycles of neoadjuvant chemotherapy preoperatively before undergoing their primary surgery for PTT. Recurrence of the disease after their first surgical treatment was reported in ten (45.4\%) patients. More specifically, the location of recurrence in these 11 patients was the thyroid bed $(n=4)$, the central compartment lymph nodes $(n=3)$, the lateral lymph nodes $(n=2)$ and the mediastinum $(n=2)$. The proportion of patients who needed reoperation after their first surgery due to recurrence, either in the thyroid bed or in regional lymph nodes was $22.7 \%$ ( $n=5$ out of 22 cases) $(1,2,4,14,16)$. Distant metastases occurred in $8(29.6 \%)$ patients; $5(62.5 \%)$ had bone metastases, 3 (37.5\%) had lung metastases and 3 (37.5\%) were diagnosed with metastases in paraaortic and clavicular lymph 


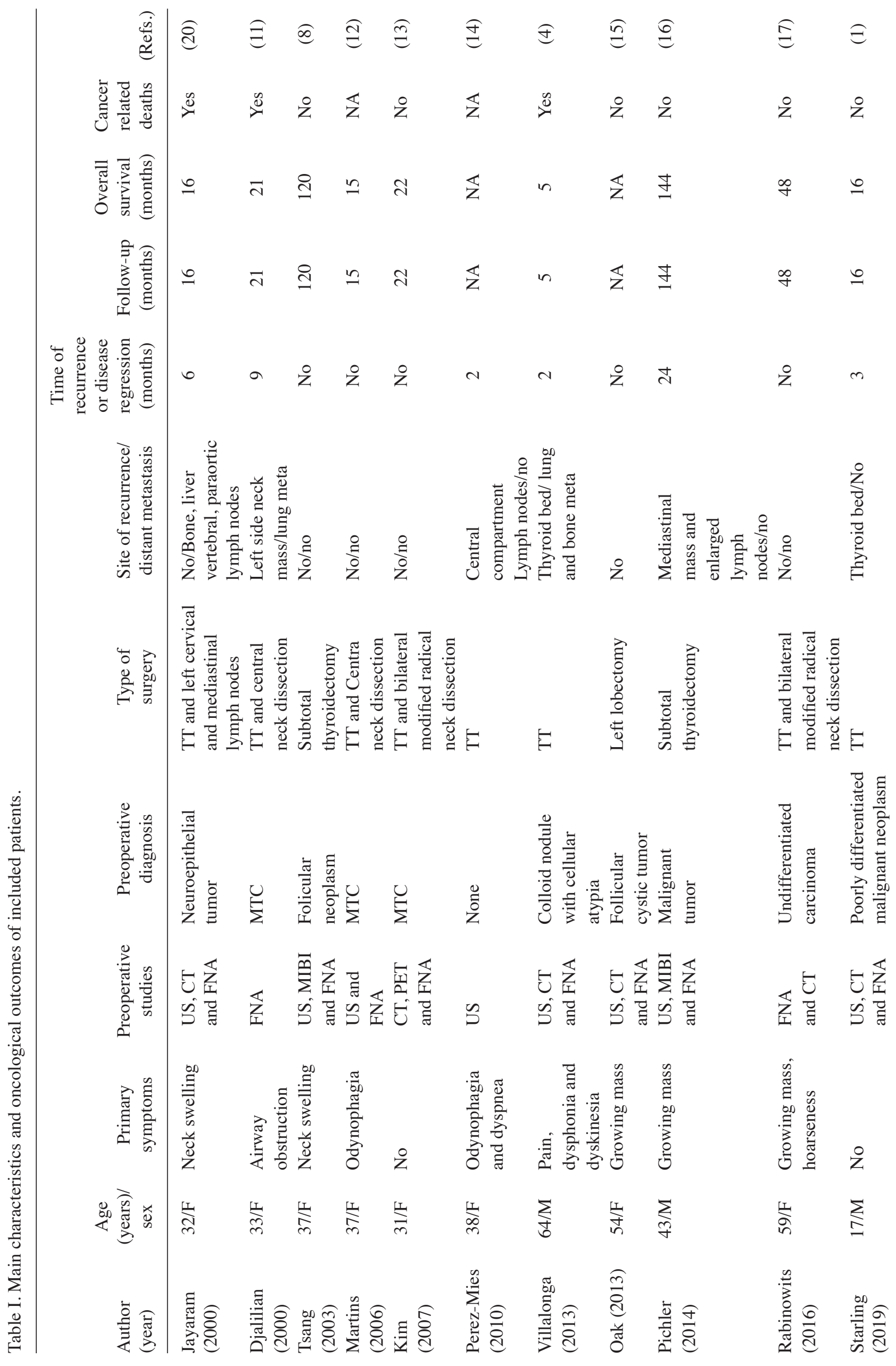




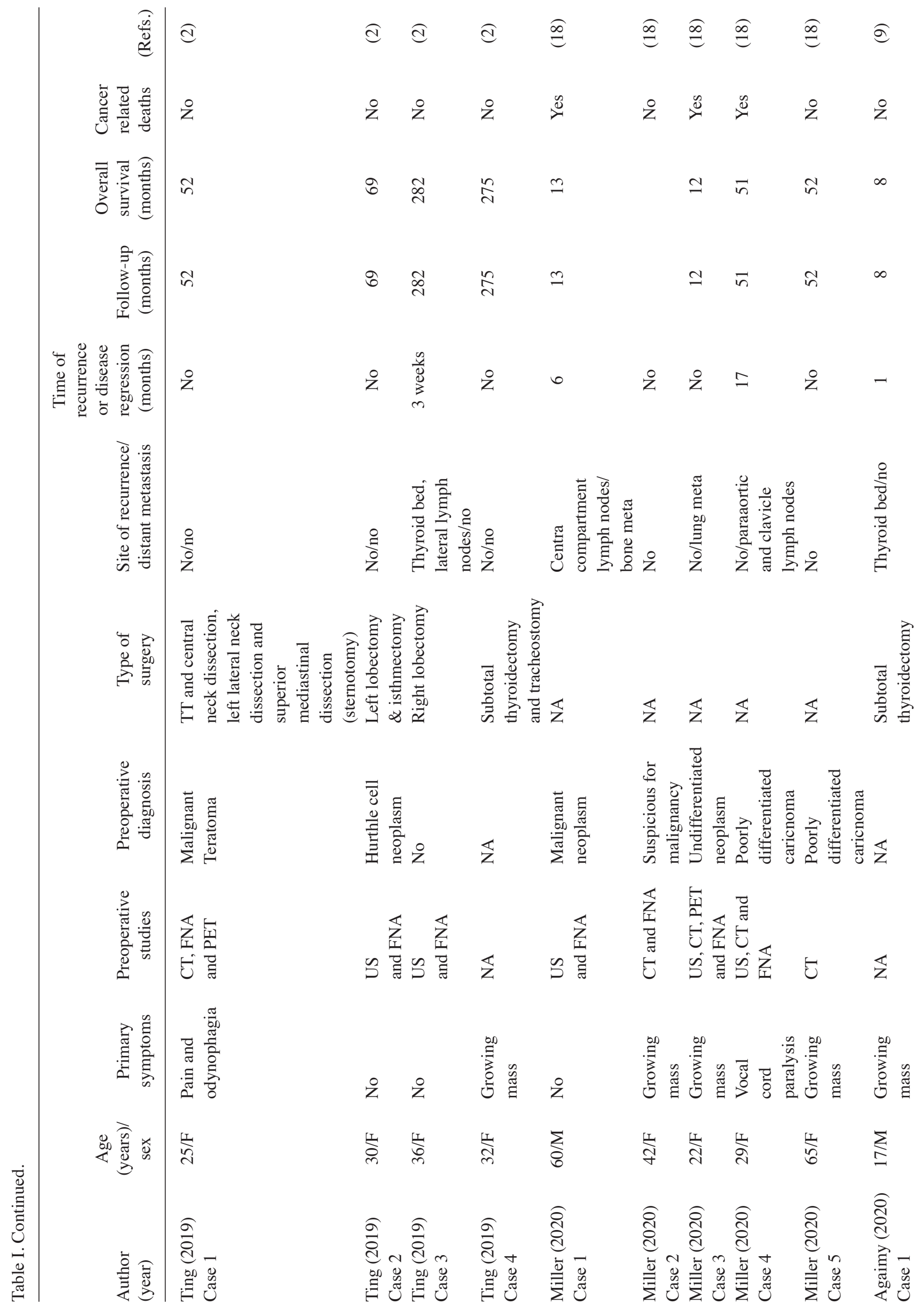




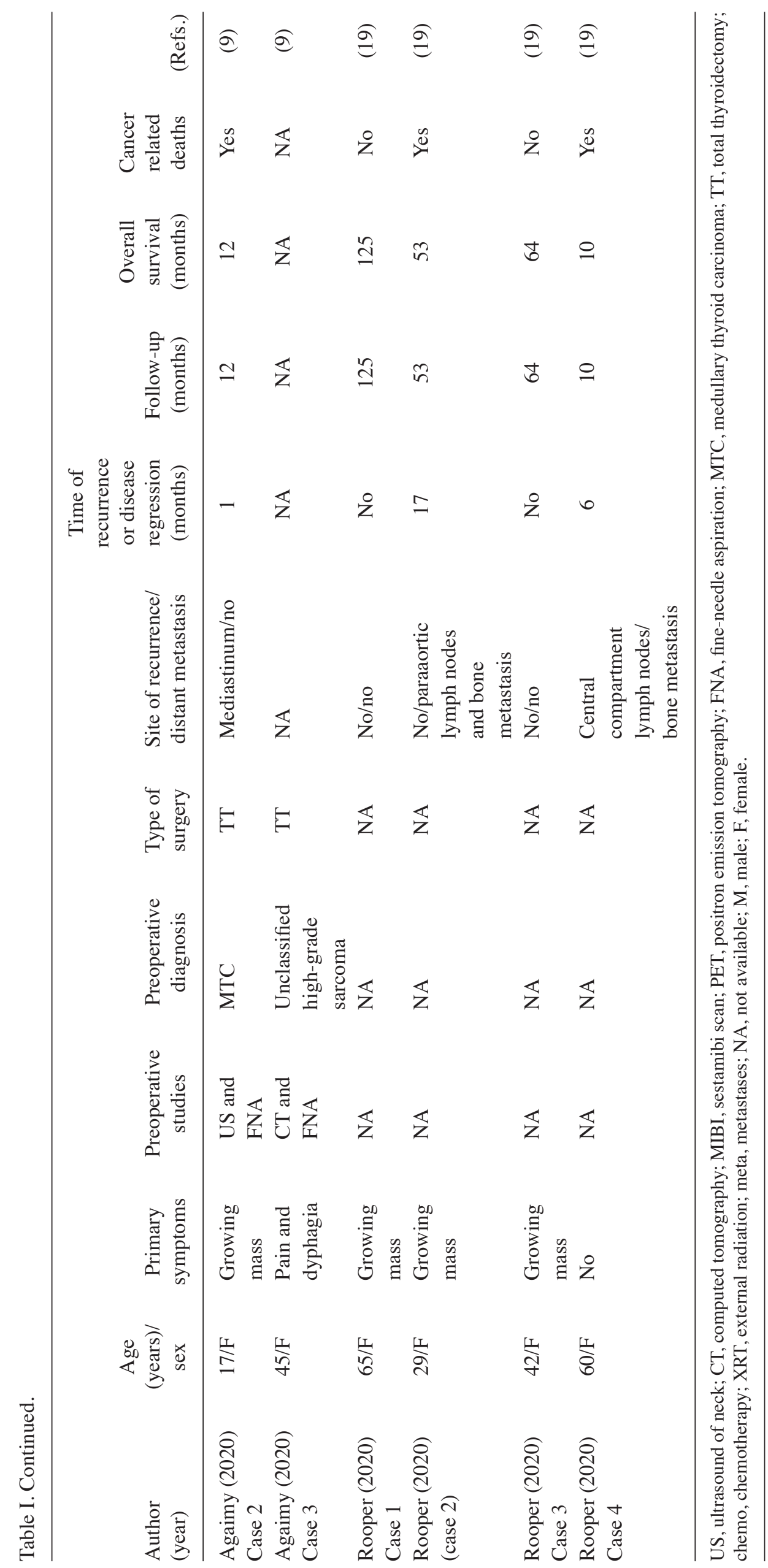


nodes $(4,11,18-20)$. The average time of recurrence or disease progression after the first surgery was 3.6 \pm 6.25 months, while the average time of follow-up was $66,7 \pm 77$ months. Nine $(39.1 \%)$ patients died due to progression of the disease $(4,9,11,18-20)$.

\section{Discussion}

In our study, we highlighted and evaluated the long-term outcomes of patients who were diagnosed with PTT and underwent surgical resection. Despite the fact that a plethora of preoperative imaging studies were selected, including US and FNA, only one patient was diagnosed with PTT preoperatively. The relatively high recurrence rates and the low average time of recurrence after the first surgery mirror the considerable aggressiveness of these rare tumors. Additionally, cancer-related death presented in almost one third of the included cases.

PTTs represent $<0.1 \%$ of all primary thyroid gland neoplasms (6). In 1908, Lurje (21) was the first author who described a case of PTT in the literature. Bale reported the first case series of PTT in the English literature in 1950 (22). One of the following criteria must be met to establish the diagnosis of PTT: i) the lesion occupies a segment of the thyroid gland, ii) the thyroid gland is totally replaced by the tumor without any evidence of thyroid gland during the surgery or in preoperative imaging modalities or iii) there is a direct connection between the thyroid gland and the tumor (6). Although PTTs are a very rare entity, they should be considered as a potential diagnosis in every young patient presenting with a high-grade thyroid tumor with a rapidly growing neck mass. The asymptomatic gradual enlargement of the neck is the most frequent clinical manifestation. Depending on the size and site of PTTs, patients can also present with significant airway obstruction (23).

Preoperative diagnosis of primary malignant thyroid teratoma is very challenging. Preoperative US usually shows a well-defined heterogenous lesion with cystic components; sensitivity and specificity of 41 and $92 \%$, respectively (23). CT and MRI of the neck could be additional preoperative diagnostic tools, presenting more favorable imaging outcomes. The typical appearance of a PTT is a well-circumscribed mass with both solid and cystic components $(24,25)$. Although, the vast majority of PTTs receive a preoperative evaluation through FNA biopsy, they are not characterized by specific and unique cytologic features. Few studies reported a neuroendocrine origin in cytologic findings of MTC (11-13). In the present systematic review, 4 patients were diagnosed with MTC and only one patient with PTT preoperatively, using a preoperative FNA biopsy (2). Moreover, regarding the 27 included patients there was no case of synchronous papillary thyroid carcinoma. Our patient had a synchronous papillary tumor, but the whole postoperative management organized according to the dominant diagnosis of primary malignant thyroid teratoma. In most cases, the final diagnosis and grading of PTT are given following a meticulous histopathological evaluation of the surgical specimen. Immature neuroepithelium is the main feature that defines malignancy in surgical specimens. Immunochemical studies can be useful to confirm neuronal differentiation and to detect ectodermal or mesodermal characteristics $(1,20,26)$. More specifically, Starling et al used several immunochemical stains, both in preoperative FNA and in final specimens (1). Immunochemical studies can also be helpful to exclude some diagnosis from our differential diagnostic algorithm. The majority of MTCs have positive calcitonin in immunochemistry, while neuroendocrine tumors are commonly keratin positive (1).

With regards to molecular characteristics of PTT, several studies have been published highlighting mutations of genes which were associated with these rare tumors $(9,17,19)$. Many authors have already reported, a DICER1 somatic mutation which potentially has a crucial role in malignant transformation in patients diagnosed with PTT. More precisely, Rabinowits et al were the first to report the presence of a pathogenic DICER1 mutation in a case of a malignant thyroid teratoma in a 59-year-old female (17). The authors linked the DICER1 mutation to the PNET-like transformation (17). In addition, Rooper et al describing pathogenicDICER1 mutations in 4 of 4 malignant PTTs, but none of 4 mature or immature PTTs (19). Although DICER1 mutations have been associated with multiple malignancies, including thyroid neoplasia (multinodular goiter, adenomas or differentiated thyroid gland neoplasia), and ovarian sex cord-stromal tumors, they have not been demonstrated in gonadal or extragonadal teratomas (9-11). The presence of the DICER1 mutation might considerably change the treatment strategy of patients with PTT, in terms of extension of surgery and neo- or adjuvant chemotherapy.

PTTs described prior to effective therapy were generally associated with an overall poor outcome and a median survival of about 8 months (1). Although, benign PTTs have an excellent prognosis after radical surgical resection, malignant PTTs have significantly poor survival even less than a year. Malignant PTTs are relatively insensitive to radiation and chemotherapy, and radical surgical approach is considered the standard of care. However, chemotherapy has been commonly used for patients with immature PTTs, producing better long-term outcomes (17). Several studies described significant long-term survival outcomes with the aggressive use of adjuvant chemotherapy, especially cis-platinum based regimens $(2,8,10)$. Although radiation therapy has often been applied in addition to surgery and chemotherapy, its role is controversial. Non-seminomatous germ-cell tumors are, generally, not sensitive in radiotherapy, and, as a result, is not clear if it is beneficial for patients diagnosed with PTT $(2,7,10)$. However, if there is an indication of invasion of the oesophagus, trachea or larynx, postoperative radiotherapy might be an advantageous option for these patients (10). Interestingly, neoadjuvant chemotherapy has been described in few cases in the current literature and may be have promising oncological outcomes in the management of patients with PTT. However, specific indications of its usage, such as extrathyroidal extension of the lesion, cervical lymph nodes metastases or distant metastases should be demonstrated $(2,17)$.

To our best of our knowledge, this present study is, the only systematic review in the literature, which describes characteristics, perioperative management and oncological outcomes of patients who were diagnosed with PTT and underwent surgical resection. Of note, the results of our systematic review should be interpreted in the context of its limitations. The total number of patients in our study is small and were meticulously selected by surgical teams, thus, objective results. Further, the true prevalence of PTT cannot be determined precisely, and data regarding their characteristics and treatment strategies is limited to case reports and small case series.Thus, further 
evaluation and research is precluded due to their rare entity. None of the included studies blinded or randomized the participants or assessors. The significant heterogeneity among the included studies and the lack of mention of certain parameters by some authors were additional limitations. Therefore, a potential selection bias must influence our results.

In conclusion, PTTs are a rare and malignant PTTs are a very aggressive tumor with a challenging preoperative diagnosis. Although radical surgery and adjuvant chemotherapy with several regimens seems to be beneficial for patients, malignant PTTs have considerably poor survival outcomes. A better and more accurate preoperative diagnosis in these rare tumors might indicate a more aggressive surgical approach should be adopted by experienced endocrine surgeons with a more extensive lymph node dissection in order to achieve better long-term outcomes.

\section{Acknowledgements}

Not applicable.

\section{Funding}

No funding was received.

\section{Availability of data and materials}

The datasets used and/or analyzed during the present study are available from the corresponding author on reasonable request.

\section{Authors' contributions}

$\mathrm{CN}$ and APa conceived and designed the study. MS, IM, KP, APs, GT and EP acquired, analyzed and interpreted the data. APa and APs drafted and revised the manuscript. $\mathrm{CN}$ and EP confirm the authenticity of all the raw data. All authors read and approved the final manuscript.

\section{Ethics approval and consent to participate}

Not applicable.

\section{Patient consent for publication}

Written informed consent was obtained from the patient for publication of this case report and any accompanying images.

\section{Competing interests}

The authors declare that they have no competing interests.

\section{References}

1. Starling CE, Sabra J, Brady B, Horton M and Traweek ST: Malignant teratoma of the thyroid: A difficult diagnosis by fine-needle aspiration. Dia Cytopathol 47: 930-934, 2019.

2. Ting J, Bell D, Ahmed S, Ying A, Waguespack SG, Tu SM, Weber R and Zafereo M: Primary Malignant thyroid teratoma: An institutional experience. Thyroid 29: 229-236, 2019.

3. Rothschild MA, Catalano P, Urken M, Brandwein M, Som P, Norton K and Biller HF: Evaluation and management of congenital cervical teratoma: Case report and review. Arch Otolaryngol Neck Surg 120: 444-448, 1994.
4. Vilallonga R, Zafon C, Ruiz-Marcellan C, Obiols G, Fort JM, Baena JA, Villanueva B, Garcia A and Sobrinho-Simões M: Malignant thyroid teratoma: Report of an aggressive tumor in a 64-year-old man. Endocr Pathol 24: 132-135, 2013.

5. Tonni G, De Felice C, Centini G and Ginanneschi C: Cervical and oral teratoma in the fetus: A systematic review of etiology, pathology, diagnosis, treatment and prognosis. Arch Gynecol Obstet 282: 355-361, 2010.

6. Thompson LD, Rosai J and Heffess CS: Primary thyroid teratomas: A clinicopathologic study of 30 cases. Cancer 88: 1149-1158, 2000.

7. Ueno NT, Amato RJ, Ro JJ and Weber RS: Primary malignant teratoma of the thyroid gland: Report and discussion of two cases. Head Neck 20: 649-653, 1998.

8. Tsang RW, Brierley JD, Asa SL and Sturgeon JF: Malignant teratoma of the thyroid: Aggressive chemoradiation therapy is required after surgery. Thyroid 13: 401-404, 2003.

9. Agaimy A, Witkowski L, Stoehr R, Cuenca JC, GonzálezMuller CA, Brütting A, Bährle M, Mantsopoulos K, Amin RM, Hartmann A, et al: Malignant teratoid tumor of the thyroid gland: An aggressive primitive multiphenotypic malignancy showing organotypical elements and frequent DICER1 alterations-is the term 'thyroblastoma' more appropriate? Virchows Arch 477: 787-798, 2020.

10. Chen JS, Lai GM and Hsueh S: Malignant thyroid teratoma of an adult: A long-term survival after chemotherapy. Am J Clin Oncol 21: 212-214, 1998.

11. Djalilian HR, Linzie B and Maisel RH: Malignant teratoma of the thyroid: Review of literature and report of a case. Am J Otolaryngol 21: 112-115, 2000.

12. Martins T, Carrilho F, Gomes L, Mesquita C, Martins MJ and Carvalheiro M: Malignant teratoma of the thyroid: Case report. Thyroid 16: 1311-1313, 2006.

13. Kim E, Tae SB, Kwon Y, Kim TH, Chung KW, Kim SW, Ro J and Lee ES: Primary malignant teratoma with a primitive neuroectodermal tumor component in thyroid gland: A case report. J Korean Med Sci 22: 568-571, 2007.

14. Pérez-Mies B, Regojo Zapata RM, García-Fernández E and Serrano MN: Malignant teratoma of the thyroid in a pregnant woman. Ann Diagn Pathol 14: 264-267, 2010.

15. Oak CY, Kim HK, Yoon TM, Lim SC, Park HB, Park HC, Han MG and Kang HC: Benign teratoma of the thyroid gland. Endocrinol Metab (Seoul) 28: 144-148, 2013.

16. Pichler R, Heidegger I, Brunner A and Steiner H: Long-term follow-up of a primary teratoma with somatic-type malignancy within the thyroid gland mimicking thyroid carcinoma. Clin Genitourin Cancer 12: e121-e124, 2014.

17. Rabinowits G, Barletta J, Sholl LM, Reche E, Lorch J and Goguen L: Successful management of a patient with malignant thyroid teratoma. Thyroid 27: 125-128, 2017.

18. Miller DL, Thompson LD, Bishop JA, Rooper LM and Ali SZ: Malignant teratomas of the thyroid gland: Clinico-radiologic and cytomorphologic features of a rare entity. J Am Soc Cytopathol 9: 221-231, 2020.

19. Rooper LM, Bynum JP, Miller KP, Lin MT, Gagan J, Thompson LD and Bishop JA: Recurrent DICER1 hotspot mutations in malignant thyroid gland teratomas: Molecular characterization and proposal for a separate classification. Am J Surg Pathol 44: 826-833, 2020.

20. Jayaram G, Cheah PL and Yip CH: Malignant teratoma of the thyroid with predominantly neuroepithelial differentiation: Fine needle aspiration cytologic, histologic and immunocytochemical features of a case. Acta Cytol 44: 375-379, 2000.

21. Lurje M: Über ein Teratom der Schilddrüse. Inaugural Disseration, J. J. Meier, Zürich, 1908.

22. Bale GF: Teratoma of the neck in the region of the thyroid gland; a review of the literature and report of 4 cases. Am J Pathol 26: $565-579,1950$.

23. Lv Z, Bai X, Sheng Q, Liu J and Wu Y: A case report of a giant mature teratoma of the thyroid gland in a young girl. Medicine (Baltimore) 98: e14703, 2019.

24. Henrichsen TL and Reading CC: Thyroid ultrasonography. Part 2: Nodules. Radiol Clin North Am 49: 417-424, 2011.

25. Wolter NE, Siegele B and Cunningham MJ: Cystic cervical teratoma: A diagnostic and management challenge. Int J Pediatr Otorhinolaryngol 95: 97-100, 2017.

26. Craver RD, Lipscomb JT, Suskind D and Velez MC: Malignant teratoma of the thyroid with primitive neuroepithelial and mesenchymal sarcomatous components. Ann Diagn Pathol 5: 285-292, 2001. 